\title{
Erratum to: Uniqueness of Embedding into a Gaussian Semigroup and a Poisson Semigroup with Determinate Jump Law on a Simply Connected Nilpotent Lie Group
}

\section{Daniel Neuenschwander}

Published online: 3 October 2009

(C) Springer Science+Business Media, LLC 2009

\section{Erratum to: J Theor Probab (2008) 21: 791-801 DOI 10.1007/s10959-008-0175-5}

There is a change in a step in the proof of the Gaussian part of Theorem 1 in the original article, and the related new proof of a result due to Pap mentioned in this paper.

In Theorem 1 in the original paper, the uniqueness property for the embedding c.c.s. among all possible c.c.s. of a Gaussian measure was asserted for simply connected nilpotent Lie groups $G$. However, the "proof" of this assertion in the latter paper contains a gap, which we will close here. In fact, the proof for the " $(2 / r)$-exponential tail decrease" of the iterated stochastic integrals under consideration in the proof of Theorem 1 in the case of several identical Brownian motions is erroneous. However, due to the skew-symmetry of the Lie brackets, this case does in fact not need to be taken into account.

The online version of the original article can be found under doi:10.1007/s10959-008-0175-5.

D. Neuenschwander $(\bowtie)$

École des Hautes Etudes Commerciales, Institut de Sciences Actuarielles, Université de Lausanne, 1015 Lausanne, Switzerland

e-mail: daniel.neuenschwander@bluewin.ch

D. Neuenschwander

Institut für mathematische Statistik und Versicherungslehre, Universität Bern, 3012 Bern,

Switzerland

D. Neuenschwander

Institut de Science Financière et d'Assurances, Université de Lyon, Université Claude Bernard Lyon 1, 50 avenue Tony Garnier, 69007 Lyon, France 
Specifically, the text in the original article

Let $Z=\int_{0}^{1} \int_{0}^{s_{m}} \ldots \int_{0}^{s_{2}} d B_{1}\left(s_{1}\right) d B_{2}\left(s_{2}\right) \ldots d B_{m}\left(s_{m}\right)$ be an iterated stochastic integral ... Using Lemma 3 and the just proved uniform $(2 / r)$-exponential tail decrease

should be replaced as follows:

Assume $\left\{B_{i}(t)\right\}_{t \geq 0}(i=1,2, \ldots, d)$ are independent one-dimensional Brownian motions such that for every $i$ it holds that $\left\{B_{i}(t)\right\}_{t \geq 0}$ is either centered or degenerate. Put

$$
Z_{d}(t):=\int_{0}^{t} \int_{0}^{s_{1}} \ldots \int_{0}^{s_{d-1}} d B_{d}\left(s_{d}\right) \ldots d B_{2}\left(s_{2}\right) d B_{1}\left(s_{1}\right) .
$$

By induction on $d$ we will first prove the assertion

$$
P\left(\sup _{0 \leq t \leq 1}\left|Z_{d}(t)\right| \geq \rho\right) \leq \exp \left(-C \rho^{2 / d}\right) \quad(\rho>0) .
$$

We will rely on the following (easy to verify) tail estimate for a one-dimensional standard normal random variable $Y$ :

$$
P(|Y| \geq \rho) \leq \exp \left(-C \rho^{2}\right) \quad(\rho>0) .
$$

We find that for an independent standard normal variable $Y$ we have

$$
\begin{aligned}
P\left(\sup _{0 \leq t \leq 1}\left|Z_{d}(t)\right| \geq \rho\right) \leq & P\left(\sup _{0 \leq t \leq 1}\left|Z_{d-1}(t)\right||Y| \geq C \rho\right) \\
\leq & P\left(\sup _{0 \leq t \leq 1}\left|Z_{d-1}(t)\right| \geq \rho^{(d-1) / d}\right)+P\left(|Y| \geq C \rho^{1 / d}\right) \\
& (\rho>0) .
\end{aligned}
$$

Now the before-mentioned assertion follows from the induction hypothesis (applied to $\left.\sup _{0 \leq t \leq 1}\left|Z_{d-1}(t)\right|\right)$ and the tail estimate for standard one-dimensional normal random variables (applied to $Y$ ). Denote by $\{W(t)\}_{t \geq 0}$ the Lévy process on $G$ with c.c.s. $\left\{\mu_{t}^{(1)}\right\}_{t \geq 0}$. Since every centered Brownian motion on the vector space $\left(\mathbb{R}^{d},+\right)$ can be written as the image of a linear endomorphism of standard Brownian motion on $\left(\mathbb{R}^{d},+\right)$, it follows that $\{W(t)\}_{t \geq 0}$ is a finite sum of processes of the type $\left\{Z_{d}(t)\right\}_{t \geq 0}$ (a priori, it would be possible that for certain $i<j$ we have that the one-dimensional Brownian motions $\left\{B_{i}(t)\right\}_{t \geq 0}$ and $\left\{B_{j}(t)\right\}_{t \geq 0}$ are both nondegenerate with $\left\{B_{i}(t)\right\}_{t \geq 0} \stackrel{a_{. s}}{=}\left\{B_{j}(t)\right\}_{t \geq 0}$; however, due to the skew-symmetry of the Lie bracket [., .], it follows that every term of this type is automatically canceled by the negative counterpart of the same term in every Lie bracket monomial). By the scaling property for the standard one-dimensional Brownian motion $\{B(t)\}_{t \geq 0}$ (which tells that the processes $\{B(u t)\}_{t \geq 0}$ and $\left\{u^{1 / 2} B(t)\right\}_{t \geq 0}$ coincide in law for $\left.u>0\right)$ this yields that there exists a constant $C>0$ such that

$$
\eta_{t}^{(1)}(\{z \in G:\|z\| \geq \rho\}) \leq \exp \left(-C \rho^{2 / d}\right) \quad(\rho>0 ; 0<t \leq 1) .
$$


By Lemma 3 and this tail estimate

Remark 1 Observe that with this change, also the independent proof of the uniqueness of the embedding Gaussian c.c.s. of a Gaussian measure on a simply connected nilpotent Lie group remains true. 